\title{
Coagulatory active constituents of Malus pumila Mill. flowers
}

\author{
Zhenhua Yin ${ }^{1,2+}$, Yong Zhang ${ }^{1 \dagger}$, Juanjuan Zhang ${ }^{1,2}$, Jinmei Wang ${ }^{3^{*}}$ and Wenyi Kang ${ }^{1,2^{*}}$ (i)
}

\begin{abstract}
Background: The flowers of Malus pumila Mill (Rosaceae) is rich in resources, but lack of medicinal value research. Chemical constituents of the same family M. pumila have coagulatory activity. Considering the coagulatory activity could be beneficial for various cardiovascular diseases, the aim of this study is to evaluate coagulatory active constituents of M. pumila flowers.

Methods: Chemical constituents of M. pumila flowers were isolated by various column chromatographies, and their coagulatory activity were evaluated by activated partial thromboplastin time (APTT), prothrombin time (PT), thrombin time (TT) and fibrinogen (FIB) in vitro.

Results: Nine compounds were isolated from M. pumila flowers, and identified as kaempferol-3-O- $\beta$-Dglucopyranoside (1), kaempferol-7-O- $\beta$-D-glucopyranoside (2), kaempferol-3-O- $\alpha$-L-arabinofuranoside (3), phloridzin (4), kaempferol (5), phloretin (6), $\beta$-sitosterol (7), lupeol (8) and pyracanthoside (9). Compounds $\mathbf{1 - 9}$ were isolated from the flowers for the first time, compounds $\mathbf{1 , 2}$ and $\mathbf{9}$ were isolated from the genus for the first time. Compound $\mathbf{2}$ could significantly shorten APTT, TT and PT, but significantly decrease the content of FIB. Compound $\mathbf{3}$ could shorten PT. Compound $\mathbf{4}$ could significantly shorten TT and PT, but significantly decrease the content of FIB. Compound $\mathbf{5}$ shortened APTT. Compound $\mathbf{6}$ and $\mathbf{7}$ could significantly shorten APTT and PT. Compound $\mathbf{9}$ was able to prolong TT and decrease the content of FIB, but shorten PT.
\end{abstract}

Conclusions: Compounds $\mathbf{2 - 7}$ possessed significant procoagulant activity in vitro, compound $\mathbf{9}$ had anticoagulant activity in vitro, which showed coagulation potential of compounds from M. pumila flowers, as a new source of bioactive molecules for therapeutic purposes.

Keywords: Malus pumila Mill, Chemical constituents, Coagulatory activity

\section{Background}

Thrombosis involves local blood clotting of the vascular system, often leading to serious health-related diseases such as heart attacks and strokes. Risk factors for thrombosis include hyperlipidemia, hyperglycemia, hypertension and cancer. These thrombotic diseases have become the leading cause of death, and incidence is increasing annually $[1,2]$. In the past few decades, drugs, including

\footnotetext{
*Correspondence: 540156686@qq.com; kangweny@hotmail.com 'Zhenhua Yin and Yong Zhang contributed equally to this work ${ }^{1}$ Zhengzhou Key Laboratory of Medicinal Resources Research, Huanghe Science and Technology College, Zhengzhou 450063, China

3 Joint International Research Laboratory of Food \& Medicine Resource

Function, Kaifeng 475004, Henan Province, China

Full list of author information is available at the end of the article
}

heparin and warfarin, have been used to prevent and treat thrombosis, but heparin is prone to spontaneous bleeding, and manifested by various mucosal hemorrhages, joint bleeding and wound bleeding. In addition, heparin-induced thrombocytopenia is a serious complication of heparin therapy $[3,4]$. Therefore, these disadvantages have necessitated a field of research aimed at discovering novel anti-thrombotic and anti-coagulant agents with fewer side effects than heparin.

Malus pumila Mill, belonging to the family Rosaceae, has been widely cultivated in around the world for centuries [5]. At present, the study of Malus pumila focused on its fruits, peels, leaves and branches. Previous research indicated that its peels contained triterpenoids, flavonoids, phenols and other components such as 
alkyl alcohols, and exhibited more potent antioxidant and antiproliferative activities [6-8]. The fruits of this plants contained flavonoids, polyphenols, glycosides, triterpenoids, steroids and fatty acid esters, and exhibited antioxidant activity [9-13].The leaf of apple has been reported to contain high levels of polyphenols, flavonoids, and exhibit great in vitro antioxidant activity and protective effect in reserpine-induced gastric ulcer in mice [14, 15]. However, chemical constituents and pharmacological effects of M. pumila flowers are still uncertain without a clear theoretical evidence.

The bioactive compounds of M. pumila flowers, may be responsible for coagulation activity, but not clear. The objective of this research was to isolate and identify the bioactive compounds of $M$. pumila flowers with potent coagulation activity, which might expand the possibility to find better coagulation drug.

\section{Methods}

\section{Plant collection}

Fresh flowers of Malus pumila were collected from Henan University Medicinal Botanical Garden in Henan Province (China) in April 2016. The specimens were authenticated and identified by Prof. Changqin Li (Henan University) according to Flora of Henan, and a voucher (specimen No: 20160410) was been deposited in the Herbarium of Huanghe Science and Technology College.

\section{Extraction and isolation}

The air-dried flowers of $M$. pumila $(700 \mathrm{~g})$ were degreased repeatedly (3 times) with petroleum ether at room temperature, 3 days each time. After petroleum ether was evaporated, the residue was extracted 3 times with $70 \%$ ethanol. The ethanol extract was concentrated under reduced pressure to give ethanol extract. The extract was evenly dispersed with an appropriate amount of water, followed by extraction with petroleum ether, ethyl acetate and $n$-butanol. The solvent was concentrated under reduced pressure to obtain petroleum ether extract $(12.0074 \mathrm{~g})$, EtOAc extract $(26.6719 \mathrm{~g})$ and $n$-butanol extract $(59.1956 \mathrm{~g})$.

The EtOAc extract was dissolved in $70 \%$ ethanol, adsorbed and loaded with D101 macroporous resin, and allowed to stand overnight, followed by gradient elution $\left(\mathrm{H}_{2} \mathrm{O}\right.$ : ethanol $\left.100 \%, 70 \%, 40 \%, 10 \%\right)$. The solvents were concentrated under reduced pressure to get water extract, $30 \%, 60 \%$ and $90 \%$ ethanol fractions.

The first and second column volumes of $60 \%$ ethanol fraction were combined and applied on a silica gel $\mathrm{H}$ using a gradient elution of $\mathrm{CH}_{2} \mathrm{Cl}_{2}$-acetone (10: 1-6:1), and then further separated by column chromatography over silica gel $\mathrm{H}$ by a gradient elution of $\mathrm{CH}_{2} \mathrm{Cl}_{2}-\mathrm{MeOH}$ (10: 1-0:1) to afford three fractions (Fr1, Fr2 and Fr3). Fr1 was chromatographed on silica gel by gradient elution with a $\mathrm{CH}_{2} \mathrm{Cl}_{2}$-acetone (4: 1-2:1) to afford two subfractions (Fr1.1 and Fr1.2). Fr1.1 (98.7 mg) was further subjected to Sephadex LH-20 (MeOH), then purified using a Gilson prepared liquid phase C18 column eluted with Acetonitrile-water containing $0.1 \%$ formic acid in water (35:65-60:40), and further purified using a Agilent SB-Phenyl column eluted with $\mathrm{MeOH}$-water containing $0.1 \%$ formic acid in water $(60: 40-90: 10 ; 50: 50)$ to yield compound $\mathbf{1}(23.0 \mathrm{mg}$ ) and compound $\mathbf{2}(5.3 \mathrm{mg})$. Fr1.2 was subjected to Sephadex LH-20 $(\mathrm{MeOH})$, and further purified using a Gilson prepared liquid phase C18 column eluted with Acetonitrile-water containing $0.1 \%$ formic acid in water (30:70-45:55) to yield compound 3 (20.3 mg). Fr3 was subjected to Sephadex LH-20 $(\mathrm{MeOH})$, and then further purified by twice preparative liquid phases to yield compound 4 (200.3 $\mathrm{mg}$ ).

The third column volume of $60 \%$ ethanol extract was subjected to silica gel (200-300 mesh) column chromatography and gradient eluted with a $\mathrm{CH}_{2} \mathrm{Cl}_{2}$-acetone (30: 1-8:1), and then was further chromatographed on silica gel $\mathrm{H}$ by gradient elution with a $\mathrm{CH}_{2} \mathrm{Cl}_{2}-\mathrm{MeOH}$ (7: 1-1:1) to afford two fractions (Fr1 and Fr 2), respectively. Fr1 was separated by column chromatography over silica gel $\mathrm{H}$ by elution with a $\mathrm{CH}_{2} \mathrm{Cl}_{2}$-acetone (10:1) to yield compound 5 (10.2 mg), and Fr 2 was applied on silica gel $\mathrm{H}$ using elution with a $\mathrm{CH}_{2} \mathrm{Cl}_{2}$-acetone (10:1) containing $30 \mu \mathrm{L}$ formic acid to afford compound $6(4.7 \mathrm{mg})$.

The first and second column volumes of $90 \%$ ethanol extract were combined together, and then was chromatographed on silica gel $\mathrm{H}$ by gradient elution with a $\mathrm{CH}_{2} \mathrm{Cl}_{2}$-acetone (40: 1-10:1), and was further purified on Sephadex $\mathrm{LH}-20\left(\mathrm{CH}_{2} \mathrm{Cl}_{2}: \mathrm{MeOH}=1: 1\right)$ to yield compound $7(5.0 \mathrm{mg})$. The third column volume of $90 \%$ ethanol extract was exposed to column chromatography on silica gel $\mathrm{H}$ eluted with $\mathrm{CH}_{2} \mathrm{Cl}_{2}$-EtOAc (20:1), then separated on Sephadex- $\mathrm{LH} 20\left(\mathrm{CH}_{2} \mathrm{Cl}_{2}: \mathrm{MeOH}=1: 1\right)$, and further purified by a semi-prepared high performance liquid phase reversed phase C18 column eluted with acetonitrile-water containing $0.1 \%$ formic acid in water (40:60-70:30) to yield compound 8 (4.7 $\mathrm{mg}$ ).

The $n-\mathrm{BuOH}$ extract was subjected to silica gel (200300 mesh) column chromatography and gradient eluted with a $\mathrm{CH}_{2} \mathrm{Cl}_{2}-\mathrm{MeOH}$ (100: 1-1:1) to give six fractions, where the second fraction was subjected to silica gel $\mathrm{H}$ eluted with a $\mathrm{CH}_{2} \mathrm{Cl}_{2}-\mathrm{MeOH}$ gradient (15:1-5:1), and then was subjected to Sephadex-LH $20(\mathrm{MeOH})$ to yield compound $9(2.4 \mathrm{mg})$. In conclusion, assigned the compound numberings for all the isolated compounds (1-9). 


\section{Coagulation time test in vitro}

The coagulation activity of compounds 1-7 and 9 were evaluated by activated partial thromboplastin time (APTT), thrombin time (TT), prothrombin time (PT), and fibrinogen (FIB) assays in vitro.

Blood samples were drawn from the auricular veins of rabbits, and the method of blood samples preparation was carried out according to Wang et al. [16]. APTT and PT were determined using the method reported by Chen et al. $[17,18]$. Briefly, for APTT assays in vitro, $50 \mu \mathrm{L}$ serum was incubated with $25 \mu \mathrm{L}$ samples and $50 \mu \mathrm{L}$ APTT assay reagent for $5 \mathrm{~min}$ at $37{ }^{\circ} \mathrm{C}$. The Clotting times were immediately recorded after the addition of $25 \mathrm{mM} \mathrm{CaCl}_{2}(100 \mu \mathrm{L})$. For PT assays in vitro, $50 \mu \mathrm{L}$ serum was incubated with $25 \mu \mathrm{L}$ samples for $3 \mathrm{~min}$ at $37^{\circ} \mathrm{C}$, and then PT assay reagent $(50 \mu \mathrm{L})$, which had been incubated at $37^{\circ} \mathrm{C}$ for $10 \mathrm{~min}$, was added to immediately recorded clotting time. Determinations of TT and FIB were performed according to the manufacturer's recommendations (Shanghai Sun Biotech Co., Ltd, China). All coagulation assays were performed in triplicates. Breviscapine $(13.33 \mathrm{mg} / \mathrm{mL})$ and Yunnanbaiyao $(40 \mathrm{mg} / \mathrm{mL})$ were used as positive controls, and the blank solvent (anhydrous ethanol: 1,2-propylene glycol: physiological saline $=1: 1: 3$, volume ratio) were used as negative controls. The concentrations of compounds 1-7 and 9 were $5 \mathrm{mg} / \mathrm{mL}$. PT, APTT, TT and FIB tests were conducted by a Semi-Automated Coagulation Analyzer (CPC Diagnostics Pvt. Ltd, India).

The results were expressed as mean $\pm \mathrm{SD}$ for three independent experiments. Numerical statistics were performed using SPSS19.0 software with single factor analysis of variance (ANOVA One-Way) to determine the difference.

\section{Results and discussion}

A total of nine compounds (1-9), were isolated from M. pumila flowers for the first time. Among them, compounds 1, 2 and 9 were isolated from the genus for the first time (Fig. 1). The chemical structures of these compounds were identified as kaempferol-3-O- $\beta$-D-glucopyranoside (1) [19], kaempferol-7-O- $\beta$-D-glucopyranoside (2) [20], kaempferol-3-O- $\alpha$-L-arabinofuranoside (3) [21],

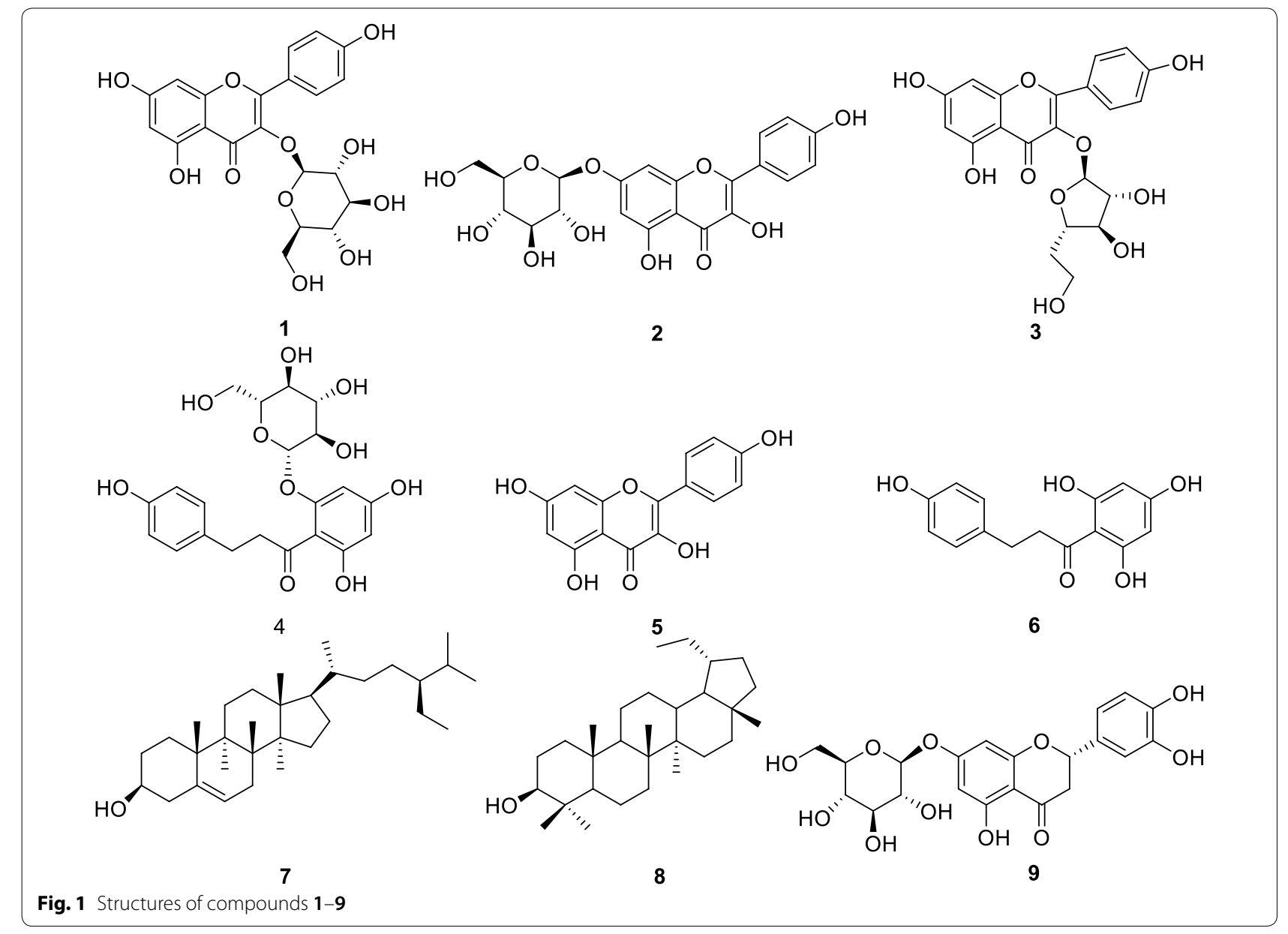


phloridzin (4) [22], kaempferol (5) [23], phloretin (6) [24], $\beta$-sitosterol (7) [25], lupeol (8) [26], pyracanthoside (9) [27].

Kaempferol-3-O- $\beta$-D-glucopyranoside (1): yellow needle crystal $(\mathrm{MeOH}), \mathrm{C}_{21} \mathrm{H}_{20} \mathrm{O}_{11}$, ESI-MS $m / z$ : 431[M$\mathrm{H}]^{+}$. ${ }^{1} \mathrm{H}-\mathrm{NMR}\left(\mathrm{DMSO}-d_{6}, 400 \mathrm{MHz}\right) \delta: 12.61(1 \mathrm{H}, \mathrm{s}$, $5-\mathrm{OH}), 8.03\left(2 \mathrm{H}, \mathrm{d}, J=8.0 \mathrm{~Hz}, \mathrm{H}-2^{\prime}, 6^{\prime}\right), 6.88(2 \mathrm{H}, \mathrm{d}$, $\left.J=8.0 \mathrm{~Hz}, \mathrm{H}-3^{\prime}, 5^{\prime}\right), 6.43(1 \mathrm{H}, \mathrm{s}, \mathrm{H}-8), 6.21(1 \mathrm{H}, \mathrm{s}, \mathrm{H}-6)$, $5.45\left(1 \mathrm{H}, \mathrm{d}, J=8.0 \mathrm{~Hz}, \mathrm{H}-1^{\prime \prime}\right) .{ }^{13} \mathrm{C}-\mathrm{NMR}$ (DMSO- $d_{6}$, $100 \mathrm{MHz}) \delta$ : 156.3 (C-2), 133.3 (C-3), 177.5 (C-4), 161.3 (C-5), 98.8 (C-6), 164.3 (C-7), 93.7 (C-8), 156.5 (C-9), $104.0(\mathrm{C}-10), 121.0\left(\mathrm{C}-1^{\prime}\right), 130.9\left(\mathrm{C}-2^{\prime}\right), 115.2\left(\mathrm{C}-3^{\prime}\right)$, $160.0\left(\mathrm{C}-4^{\prime}\right), 115.2\left(\mathrm{C}-5^{\prime}\right), 130.9\left(\mathrm{C}-6^{\prime}\right), 101.0\left(\mathrm{C}-1^{\prime \prime}\right), 74.3$ (C-2"), $76.5\left(\mathrm{C}-3^{\prime \prime}\right), 70.0\left(\mathrm{C}-4^{\prime \prime}\right), 77.5\left(\mathrm{C}-5^{\prime \prime}\right), 60.9\left(\mathrm{C}-6^{\prime \prime}\right)$.

Kaempferol-7- $O-\beta-D$-glucopyranoside (2): yellow pow$\operatorname{der}(\mathrm{MeOH}), \mathrm{C}_{21} \mathrm{H}_{20} \mathrm{O}_{11}$, ESI-MS $m / z: 449[\mathrm{M}+\mathrm{H}]^{+} .{ }^{1} \mathrm{H}-$ NMR (DMSO- $\left.d_{6}, 400 \mathrm{MHz}\right) \delta: 8.05(2 \mathrm{H}, \mathrm{d}, J=8.0 \mathrm{~Hz}$, $\left.\mathrm{H}-2^{\prime}, 6^{\prime}\right), 6.92\left(2 \mathrm{H}, \mathrm{d}, J=8.0 \mathrm{~Hz}, \mathrm{H}-3^{\prime}, 5^{\prime}\right), 6.75(1 \mathrm{H}, \mathrm{s}$, $\mathrm{H}-8), 6.41(1 \mathrm{H}, \mathrm{s}, \mathrm{H}-6), 5.10\left(1 \mathrm{H}, \mathrm{s}, \mathrm{H}-1^{\prime \prime}\right) .{ }^{13} \mathrm{C}-\mathrm{NMR}$ (DMSO- $\left.d_{6}, 100 \mathrm{MHz}\right) \delta: 147.4(\mathrm{C}-2), 135.5$ (C-3), 175.7 (C-4), 160.3 (C-5), 98.8 (C-6), 162.3 (C-7), 94.3 (C-8), 155.8 (C-9), 104.5 (C-10), $121.3\left(\mathrm{C}-1^{\prime}\right), 129.8\left(\mathrm{C}-2^{\prime}\right)$, $115.5\left(\mathrm{C}-3^{\prime}\right), 160.0\left(\mathrm{C}-4^{\prime}\right), 115.5\left(\mathrm{C}-5^{\prime}\right), 129.8\left(\mathrm{C}-6^{\prime}\right), 100.1$ $\left(\mathrm{C}-1^{\prime \prime}\right), 73.2\left(\mathrm{C}-2^{\prime \prime}\right), 77.2\left(\mathrm{C}-3^{\prime \prime}\right), 69.4\left(\mathrm{C}-4^{\prime \prime}\right), 76.5\left(\mathrm{C}-5^{\prime \prime}\right)$, $60.7\left(\mathrm{C}-6^{\prime \prime}\right)$.

Kaempferol-3- $O-\alpha$-L-arabinofuranoside (3): yellow powder (MeOH), $\mathrm{C}_{20} \mathrm{H}_{18} \mathrm{O}_{10}$, ESI-MS $m / z: 419[\mathrm{M}+\mathrm{H}]^{+}$. ${ }^{1} \mathrm{H}-\mathrm{NMR}\left(\mathrm{DMSO}-d_{6}, 400 \mathrm{MHz}\right) \delta: 12.63(1 \mathrm{H}, \mathrm{s}, 5-\mathrm{OH})$, $8.02\left(2 \mathrm{H}, \mathrm{d}, J=8.0 \mathrm{~Hz}, \mathrm{H}-2^{\prime}, 6^{\prime}\right), 6.89(2 \mathrm{H}, \mathrm{d}, J=8.0 \mathrm{~Hz}$, $\left.\mathrm{H}-3^{\prime}, 5^{\prime}\right), 6.45(1 \mathrm{H}, \mathrm{s}, \mathrm{H}-8), 6.21(1 \mathrm{H}, \mathrm{s}, \mathrm{H}-6), 5.63(1 \mathrm{H}, \mathrm{s}$, $\left.\mathrm{H}-1^{\prime \prime}\right) .{ }^{13} \mathrm{C}-\mathrm{NMR}$ (DMSO- $\left.d_{6}, 100 \mathrm{MHz}\right) \delta: 156.4(\mathrm{C}-2)$, 133.5 (C-3), 177.7 (C-4), 161.3 (C-5), 98.8 (C-6), 164.3 (C-7), 93.4 (C-8), 156.9 (C-9), 104.1 (C-10), $120.8\left(\mathrm{C}-1^{\prime}\right)$, $130.8\left(\mathrm{C}-2^{\prime}\right), 115.5\left(\mathrm{C}-3^{\prime}\right), 160.0\left(\mathrm{C}-4^{\prime}\right), 115.5\left(\mathrm{C}-5^{\prime}\right), 130.8$ $\left(\mathrm{C}-6^{\prime}\right), 108.1\left(\mathrm{C}-1^{\prime \prime}\right), 82.2\left(\mathrm{C}-2^{\prime \prime}\right), 77.2\left(\mathrm{C}-3^{\prime \prime}\right), 86.4\left(\mathrm{C}-4^{\prime \prime}\right)$, $61.0\left(\mathrm{C}-5^{\prime \prime}\right)$.

Phloridzin (4): white needle crystal (MeOH), mp. 168$169{ }^{\circ} \mathrm{C}, \mathrm{C}_{21} \mathrm{H}_{24} \mathrm{O}_{10}$, ESI-MS $m / z: 437[\mathrm{M}+\mathrm{H}]^{+} .{ }^{1} \mathrm{H}-\mathrm{NMR}$ (DMSO- $\left.d_{6}, 400 \mathrm{MHz}\right) \delta: 7.03(2 \mathrm{H}, \mathrm{d}, J=8.0 \mathrm{~Hz}, \mathrm{H}-3,5)$, $6.63(2 \mathrm{H}, \mathrm{d}, J=8.0 \mathrm{~Hz}, \mathrm{H}-2,6), 6.13\left(1 \mathrm{H}, \mathrm{s}, \mathrm{H}-5^{\prime}\right), 5.93$ $\left(1 \mathrm{H}, \mathrm{d}, J=4.0 \mathrm{~Hz}, \mathrm{H}-3^{\prime}\right), 5.33\left(1 \mathrm{H}, \mathrm{d}, J=4.0 \mathrm{~Hz}, \mathrm{OH}-4^{\prime \prime}\right)$, $5.19\left(1 \mathrm{H}, \mathrm{d}, J=4.0 \mathrm{~Hz}, \mathrm{OH}-3^{\prime \prime}\right), 5.09(1 \mathrm{H}, \mathrm{d}, J=4.0 \mathrm{~Hz}$, $\left.\mathrm{OH}-2^{\prime \prime}\right), 4.93\left(1 \mathrm{H}, \mathrm{d}, J=8.0 \mathrm{~Hz}, \mathrm{H}-1^{\prime \prime}\right), 4.63(1 \mathrm{H}, \mathrm{t}$, $\left.J=8.0,4.0 \mathrm{~Hz}, \mathrm{OH}-6^{\prime \prime}\right), 3.71\left(1 \mathrm{H}, \mathrm{m}, \mathrm{H}-6^{\prime \prime}\right), 3.19(1 \mathrm{H}, \mathrm{t}$, $\left.J=8.4 \mathrm{~Hz}, \mathrm{H}-2^{\prime \prime}\right), 2.78(2 \mathrm{H}, \mathrm{t}, J=8.0,8.0 \mathrm{~Hz}, \mathrm{H}-\beta) .{ }^{13} \mathrm{C}-$ NMR (DMSO- $\left.d_{6}, 100 \mathrm{MHz}\right) \delta: 29.0(\mathrm{C}-\beta), 45.0(\mathrm{C}-\alpha)$, $204.7(C=O), 131.6(C-1), 129.2(C-2,6), 115.0(C-3,5)$, 155.3 (C-4), $105.2\left(\mathrm{C}-1^{\prime}\right), 165.4\left(\mathrm{C}-2^{\prime}\right), 96.9\left(\mathrm{C}-3^{\prime}\right), 164.6$ (C-4'), 94.4 (C-5'), $160.8\left(\mathrm{C}-6^{\prime}\right), 100.8\left(\mathrm{C}-1^{\prime \prime}\right), 73.2\left(\mathrm{C}-2^{\prime \prime}\right)$, $77.3\left(\mathrm{C}-3^{\prime \prime}\right), 69.5\left(\mathrm{C}-4^{\prime \prime}\right), 76.7\left(\mathrm{C}-5^{\prime \prime}\right), 60.6\left(\mathrm{C}-6^{\prime \prime}\right)$.

Kaempferol (5): yellow powder $(\mathrm{MeOH}), \mathrm{C}_{15} \mathrm{H}_{10} \mathrm{O}_{6}$, ESI-MS $m / z: 287[\mathrm{M}+\mathrm{H}]^{+} .{ }^{1} \mathrm{H}-\mathrm{NMR}(\mathrm{MeOH}, 400 \mathrm{MHz})$ $\delta: 8.08\left(2 \mathrm{H}, \mathrm{d}, J=6.4 \mathrm{~Hz}, \mathrm{H}-2^{\prime}, 6^{\prime}\right), 6.89(2 \mathrm{H}, \mathrm{d}, J=7.6 \mathrm{~Hz}$,
$\left.\mathrm{H}-3^{\prime}, 5^{\prime}\right), 6.39(1 \mathrm{H}, \mathrm{s}, \mathrm{H}-8), 6.18(1 \mathrm{H}, \mathrm{s}, \mathrm{H}-6) .{ }^{13} \mathrm{C}-\mathrm{NMR}$ (100 MHz, MeOH) $\delta: 146.8$ (C-2), 135.6 (C-3), 175.9 (C-4), 156.2 (C-5),99.3 (C-6), 162.5 (C-7), 94.4 (C-8),

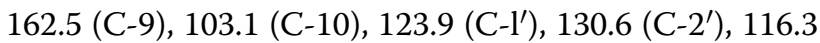
(C-3'), 160.4 (C-4'), $116.3\left(\mathrm{C}-5^{\prime}\right), 130.6\left(\mathrm{C}-6^{\prime}\right)$.

Phloretin (6): white powder (MeOH-water), $\mathrm{C}_{15} \mathrm{H}_{14} \mathrm{O}_{5}$. ${ }^{1} \mathrm{H}-\mathrm{NMR}\left(\mathrm{DMSO}-d_{6}, 400 \mathrm{MHz}\right) \delta: 7.02(2 \mathrm{H}, \mathrm{d}, J=7.6 \mathrm{~Hz}$, $\mathrm{H}-2,6), 6.66(2 \mathrm{H}, \mathrm{d}, J=7.6 \mathrm{~Hz}, \mathrm{H}-3,5), 5.76(2 \mathrm{H}$, brs, $\left.\mathrm{H}-3^{\prime}, 5^{\prime}\right), 3.21(2 \mathrm{H}, \mathrm{t}, J=7.6 \mathrm{~Hz}, \mathrm{H}-\alpha), 2.75(2 \mathrm{H}, \mathrm{t}$, $J=7.4 \mathrm{~Hz}, \mathrm{H}-\beta) .{ }^{13} \mathrm{C}-\mathrm{NMR}\left(100 \mathrm{MHz}\right.$, DMSO- $\left.d_{6}\right) \delta$ : 132.2 (C-1), 130.1 (C-2, 6), 115.5 (C-3, 5), 155.9 (C-4), 204.4 $(\mathrm{C}=\mathrm{O}), 104.2\left(\mathrm{C}-1^{\prime}\right), 165.4\left(\mathrm{C}-2^{\prime}, 6^{\prime}\right), 95.1\left(\mathrm{C}-3^{\prime}, 5^{\prime}\right)$, $165.9\left(\mathrm{C}-4^{\prime}\right), 45.8(\mathrm{C}-\alpha), 30.1(\mathrm{C}-\beta)$.

$\beta$-Sitosterol (7): white needle crystal (acetone), $\mathrm{C}_{29} \mathrm{H}_{50} \mathrm{O}, \mathrm{mp} 136-137{ }^{\circ} \mathrm{C}$, EI-MS $m / z: 414.4[\mathrm{M}]^{+} .{ }^{1} \mathrm{H}-$ NMR $\left(\mathrm{CDCl}_{3}, 400 \mathrm{MHz}\right) \delta: 5.36(1 \mathrm{H}$, brs, $\mathrm{H}-6), 3.52(1 \mathrm{H}$, $\mathrm{m}, \mathrm{H}-3 \alpha), 0.68\left(3 \mathrm{H}, \mathrm{s}, \mathrm{CH}_{3}-18\right), 0.80\left(3 \mathrm{H}, \mathrm{s}, \mathrm{CH}_{3}-27\right), 0.82$ $\left(3 \mathrm{H}, \mathrm{d}, J=8 \mathrm{~Hz}, \mathrm{CH}_{3}-26\right), 0.86\left(3 \mathrm{H}, \mathrm{s}, \mathrm{CH}_{3}-29\right), 0.93(3 \mathrm{H}$, d, $\left.J=8 \mathrm{~Hz}, \mathrm{CH}_{3}-21\right), 1.01\left(3 \mathrm{H}, \mathrm{s}, \mathrm{CH}_{3}-19\right)$.

Lupeol (8): white needle crystal (chloroform-acetone), mp 214-217 ${ }^{\circ} \mathrm{C} . \mathrm{C}_{30} \mathrm{H}_{50} \mathrm{O}$. EI-MS $m / z: 426[\mathrm{M}]^{+} .{ }^{1} \mathrm{H}-\mathrm{NMR}$ $\left(\mathrm{CDCl}_{3}, 400 \mathrm{MHz}\right) \delta: 4.69(1 \mathrm{H}$, brs, $\mathrm{H}-29), 4.57(1 \mathrm{H}$, brs, $\mathrm{H}-29), 3.19(1 \mathrm{H}, \mathrm{dd}, J=11.2,5.2 \mathrm{~Hz}, \mathrm{H}-3), 2.38(1 \mathrm{H}, \mathrm{m}$, H-19), 1.68 (3H, s, H-30), 1.03 (3H, s, H-26), 0.97 (3H, s, $\mathrm{H}-23), 0.95$ (3H, s, H-27), 0.83 (3H, s, H-25), 0.79 (3H, s, $\mathrm{H}-28), 0.76$ (3H, s, H-24). ${ }^{13} \mathrm{C}-\mathrm{NMR}\left(\mathrm{CDCl}_{3}, 100 \mathrm{MHz}\right) \delta$ : 38.9 (C-1), 27.7 (C-2), 79.2 (C-3), 39.1 (C-4), 55.5 (C-5), 18.5 (C-6), 34.5 (C-7), 41.1 (C-8), 50.7 (C-9), 37.4 (C-10), 21.2 (C-11), 25.4 (C-12), 38.3 (C-13), 43.0 (C-14), 27.6 (C-15), 35.8 (C-16), 43.2 (C-17), 48.2 (C-18), 48.6 (C-19), 151.1 (C-20), 30.1 (C-21), 40.2 (C-22), 28.2 (C-23), 15.5 (C-24), 16.3 (C-25), 16.2 (C-26), 14.7 (C-27), 18.2 (C-28), 109.5 (C-29), 19.5 (C-30).

Pyracanthoside (9): light yellow powder $(\mathrm{MeOH})$, $\mathrm{C}_{21} \mathrm{H}_{22} \mathrm{O}_{11}$, ESI-MS $m / z: 449[\mathrm{M}-\mathrm{H}]^{-}, 287[\mathrm{M}-\mathrm{H}-\mathrm{Glc}]^{-}$. ${ }^{1} \mathrm{H}-\mathrm{NMR}(\mathrm{MeOH}, 400 \mathrm{MHz}) \delta: 2.62(1 \mathrm{H}, \mathrm{d}, J=5.8 \mathrm{~Hz}$, $\mathrm{H}-3 \alpha), 2.96(1 \mathrm{H}, \mathrm{d}, J=2.6 \mathrm{~Hz}, \mathrm{H}-3 \beta), 4.75(1 \mathrm{H}, \mathrm{d}$, $\left.J=6.4 \mathrm{~Hz}, \mathrm{H}-1^{\prime \prime}\right), 5.32(1 \mathrm{H}, \mathrm{d}, J=5.8 \mathrm{~Hz}, \mathrm{H}-3 \alpha), 6.38$ $(1 \mathrm{H}, \mathrm{s}, \mathrm{H}-8), 6.85\left(1 \mathrm{H}, \mathrm{d}, J=9.4 \mathrm{~Hz}, \mathrm{H}-5^{\prime}\right), 7.46(1 \mathrm{H}, \mathrm{dd}$, $\left.J=1.8,9.4 \mathrm{~Hz}, \mathrm{H}-6^{\prime}\right), 7.50\left(1 \mathrm{H}, \mathrm{d}, J=1.8 \mathrm{~Hz}, \mathrm{H}-2^{\prime}\right) .{ }^{13} \mathrm{C}-$ NMR (100 MHz, MeOH) $\delta: 78.8$ (C-2), 42.2 (C-3), 197.2 (C-4), 163.0 (C-5),96.5 (C-6), 165.3 (C-7), 95.5 (C-8),

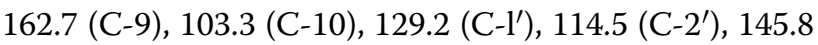
(C-3'), 145.2 (C-4'), 115.4 (C-5'), $118.2\left(\mathrm{C}-6^{\prime}\right), 99.6\left(\mathrm{C}-1^{\prime \prime}\right)$, $73.0\left(\mathrm{C}-2^{\prime \prime}\right), 76.3\left(\mathrm{C}-3^{\prime \prime}\right), 69.5\left(\mathrm{C}-4^{\prime \prime}\right), 77.1\left(\mathrm{C}-5^{\prime \prime}\right), 60.6$ $\left(\mathrm{C}-6^{\prime \prime}\right)$.

Flavonoids, steroids and terpenes were isolated from the flowers of M. pumila. Several secondary metabolites from plants that present many important biological activities, such as anticoagulant and antioxidant [28], some play a important role in triggering cardiovascular activities. Cao et al. [29] reported that flavonoids such as acacetin and tilianin exhibited significant anticoagulant 
activity through prolonging $\mathrm{PT}, \mathrm{APTT}$, TT and reducing FIB content, and Xie et al. [25] found terpenes such as ursolic acid, oleanolic acid and suavissimoside R1 exhibited anticoagulant activity in vitro. These findings prompted us to study the coagulatory active constituents from flowers of $M$. pumila, seeking new therapeutic purposes for this plant.

The result of coagulation activity of compounds 1-7 and 9 isolated from flowers were showed in Fig. 2a. In APTT test, compared with blank group, compounds 2 and 5-7 were able to significantly shorten the clotting time ( $p<0.001$ or $p<0.01$ ), and the effect of compounds 6 and 7 was significantly better than that of Yunnanbaiyao as positive control $(p<0.01)$, demonstrating their procoagulant activity.

As can be observed in Fig. 2b, in TT test, compared with blank group, compounds $\mathbf{2}$ and $\mathbf{4}$ could be significantly shorten the clotting time $(p<0.001)$, and was significantly better than Yunnanbaiyao $(p<0.001)$, while compounds 6 and 9 significantly prolonged the clotting time compared with blank group $(p<0.001$ or $p<0.05)$, and the effect was weaker than breviscapine as positive control, and had statistical significance $(p<0.001)$.

As can be observed in Fig. 2c, in PT test, compounds 2-4, 6-7 and 9 could significantly shorten clotting time compared with blank group $(p<0.001)$, and the effect was weaker than Yunnanbaiyao, and had statistical significance $(p<0.001)$.

As can be observed in Fig. 2d, in FIB test, compared with blank group, compounds $2-4$ and 9 could significantly decrease the FIB content $(p<0.001$ or $p<0.05)$, and the effect of compounds 2-4 was significantly weaker than Yunnanbaiyao $(p<0.001)$, compound 9 was equal to that of Yunnanbaiyao $(p>0.05)$, compound 1 could significantly increase the FIB content compared with blank group $(p<0.01)$, and was significantly weaker than breviscapine $(p<0.001)$.

Normally, 12 blood coagulatory factors are sequentially combined with coagulation process, which include the intrinsic and/or common pathway and extrinsic pathway [30]. In clinical tests of blood coagulation, several wellestablished analyses are used to indicate coagulation activity including APTT, PT, TT and FIB. Normally, PT is
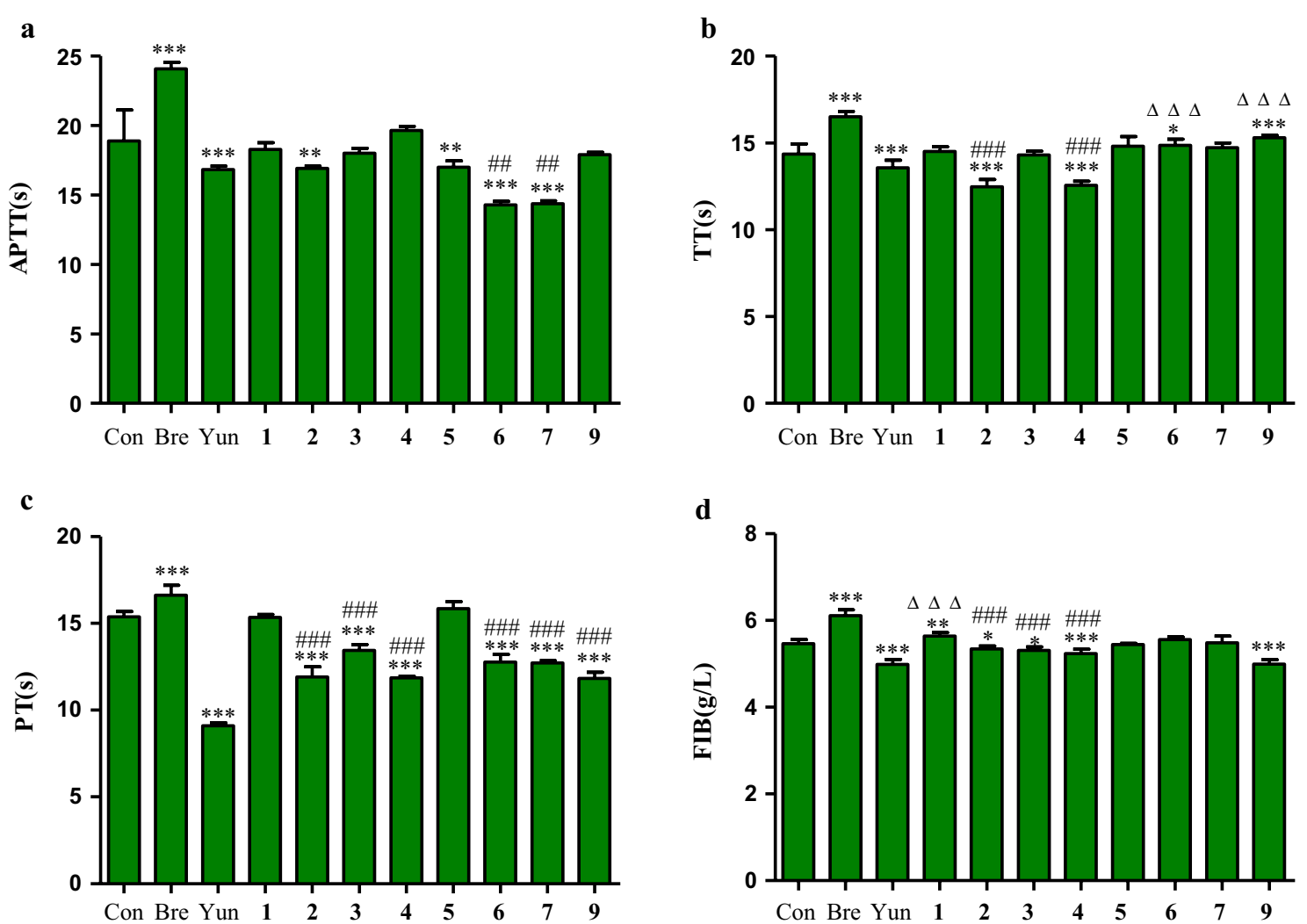

Fig. 2 Effects of compounds $\mathbf{1 - 7}$ and $\mathbf{9}$ on plasma coagulation parameters in vitro (a APPT; $\mathbf{b} P \mathrm{PT} ; \mathbf{c T T} ; \mathbf{d}$ FIB. $\mathrm{n}=6$ ). Compared with control group (Con), ${ }^{* * *} p<0.001<{ }^{* *} p<0.01<{ }^{*} p<0.05$; Compared with Yunnan Baiyao (Yun), ${ }^{\# \#} p<0.001<{ }^{\# \#} p<0.01<\# p<0.05$; Compared with breviscapine (Bre), $\triangle \triangle_{p}<0.001<\Delta_{p}<0.01<\Delta_{p}<0.05$ 
used to evaluate the overall efficiency of extrinsic clotting pathway, a prolonged PT indicates a deficiency in coagulation factors V, VII and X. On the other hand, APTT is a test of the intrinsic clotting activity, a prolonged APTT usually represents a deficiency in factors VIII, IX, XI, XII and VonWillebrand's factor. Whereas prolongation of TT indicates inhibition of thrombin activity or polymerization, FIB mainly reflects the content of fibrinogen [25, $31,32]$. In this study, compound $\mathbf{2}$ could significantly shorten APTT, TT and PT, but significantly decrease the content of FIB, it could therefore be speculated that its procoagulant activity might be related to the changes of coagulation factors in both extrinsic and intrinsic clotting pathways. For compound 3, shortening PT demonstrated inhibition of the extrinsic pathway. Compound $\mathbf{4}$ could significantly shorten TT and PT, but significantly decrease the content of FIB, demonstrating the changes of coagulation factor activity in exogenous coagulation pathways caused its procoagulant activity. For compound 5, shortening APTT demonstrated inhibition of the intrinsic clotting pathway. Compound $\mathbf{6}$ and 7 could significantly shorten APTT and PT, demonstrating their procoagulant activity might result from altered activity of coagulation factors in both extrinsic and intrinsic clotting pathways. Compound 9 was able to prolong TT and decrease the content of FIB, but shorten PT, demonstrating its anticoagulant activity might be related to activation of thrombin activity.

In conclusion, the presented results showed that compounds 2-7 had significant beneficial effects as a procoagulant agent, compound 9 had significant beneficial effects as an anticoagulant agent. Thus, this study showed the potential of $M$. pumila flowers as a new source of bioactive molecules for therapeutic purposes. Up to now, many scholars have paid attention to the structure-activity relationship of active natural products and modified their structures, for example, Reddy et al. [33-35] had modified the natural products with antioxidant properties and antiproliferative activities, which would guide us to further study on compounds $2-7$ and 9 .

\section{Conclusion}

Nine compounds were isolated from the flowers of $M$. pumila, and identified as kaempferol-3-O- $\beta$-Dglucopyranoside, kaempferol-7-O- $\beta$-D-glucopyranoside, kaempferol-3-O- $\alpha$-L-arabinofuranoside, phloridzin, kaempferol, phloretin, $\beta$-sitosterol, lupeol and pyracanthoside. All compounds were isolated from the flowers for the first time, compounds 1, 2 and 9 were isolated from the genus for the first time. Compounds 2-7 had procoagulant activity, compound 9 had anticoagulant activity. Since compounds with procoagulant or anticoagulant that could be used in current medicine for treatment of various cardiovascular diseases, we suggested that the flowers of M. pumila showed promising potential as a future therapeutic agent. Thus, the results presented herein might provide scientific evidence, at least, for the widespread use of this plant in complementary and alternative drugs and to demonstrate its potential as a new source of bioactive molecules for therapeutic purposes.

\section{Abbreviations \\ ATPP: activated partial thromboplastin time; TT: thrombin time; PT: pro- thrombin time; FIB: fbrinogen; Con: control group; Yun: Yunnan Baiyao; Bre: breviscapine (Bre).}

\section{Authors' contributions}

$Z H Y, Y Z$ and $W Y K$ conceived and designed the experiments, $Z H Y$ and $Y Z$ made signifcant contributions to design, experimentation, acquisition and interpretation of data and writing of manuscript,and contributed equally to this work. JJZ participated in extraction and isolation, JMW participated in coagulatory activity experiment, extraction and MS revised the manuscript for important intellectual content. WYK supervised the study and reviewed the manuscript. All authors read and approved the final manuscript.

\section{Author details}

${ }^{1}$ Zhengzhou Key Laboratory of Medicinal Resources Research, Huanghe Science and Technology College, Zhengzhou 450063, China. ${ }^{2}$ Henan Joint International Research Laboratory of Drug Discovery of Small Molecules, Zhengzhou 450063, China. ${ }^{3}$ Joint International Research Laboratory of Food \& Medicine Resource Function, Kaifeng 475004, Henan Province, China.

\section{Competing interests}

The authors declare that they have no competing interests.

\section{Availability of data and materials}

The content of this manuscript was argued and are useful on requirement from the corresponding author.

\section{Funding}

This work was supported by Henan Province University Science and Technology Innovation Team (16IRTSTHN019), Key Project in Science and Technology of Henan Province (182102110473) and Key Research Projects of Colleges and Universities in Henan province (18A360019).

\section{Publisher's Note}

Springer Nature remains neutral with regard to jurisdictional claims in published maps and institutional affiliations.

Received: 27 March 2018 Accepted: 13 November 2018 Published online: 03 December 2018

\section{References}

1. Leopold JA, Loscalzo J (2009) Oxidative risk for atherothrombotic cardiovascular disease. Free Radic Biol Med 47(12):1673-1706

2. Suleria HAR, Masci PP, Zhao KN, Addepalli R, Chen W, Osborne SA, Gobe GC (2017) In vitro anti-thrombotic activity of extracts from blacklip abalone (Haliotis rubra) processing waste. Mar Drugs 15(1):240

3. Franchini M, Liumbruno GM, Bonfanti C, Lippi G (2016) The evolution of anticoagulanttherapy. Blood Transfus 14(2):175-184

4. Ahmed I, Majeed A, Powell R (2007) Heparin induced thrombocytopenia: diagnosis and management update. Postgrad Med J 83(983):575-582

5. Editorial Board of Chinese Flora of Chinese Academy of Sciences (1974) Chinese Flora. Sci Press 36: 380 
6. He AJ, Liu RH (2007) Triterpenoids isolated from apple peels have potent antiproliferative activity and may be partially responsible for apple's anticancer activity. J Agric Food Chem 55(11):4366-4370

7. He AJ, Liu RH (2008) Phytochemicals of apple peels: isolation, structure elucidation, and their antiproliferative and antioxidant activities. J Agric Food Chem 56(21):9905-9910

8. Lister CE, Lancaster JE, Sutton KH, Walker JRL (1994) Developmental changes in the concentration and composition of flavonoids in skin of a red and a green apple cultivar. J Sci Food Agric 64(2):155-161

9. Ceymann M, Arrigoni E, Schärer H, Baumgartner D, Nising AB, Hurrell RF (2011) Rapid high performance screening method using UHPLC-MS to quantify 12 polyphenol compounds in fresh apples. Anal Methods 3(8):1774-1778

10. Schwab W, Schreier P (1990) Vomifoliol 1-O- $\beta$-D-xylopyranosyl-6-O- $\beta$-Dglucopyranoside: a disaccharide glycoside from apple fruit. Phytochemistry 29(1):161-164

11. Herderich M, Feser W, Schreier P (1992) Vomifoliol 9-O-beta-D-glucopyranosyl-4-O-beta-D-xylopyranosyl-6-O-beta-D-glucopyranoside: a trisaccharide glycoside from apple fruit. Phytochemistry 31(3):895-897

12. Cefarelli G, Dabrosca B, Fiorentino A, Izzo A, Mastellone C, Pacifico S, Piscopo V (2006) Free-radical-scavenging and antioxidant activities of secondary metabolites from reddened cv. Annurca apple fruits. J Agric Food Chem 54(3):803-809

13. Cefarelli G, Abrosca BD, Fiorention A, Izzo A, Monaco P (2005) Isolation, characterization, and antioxidant activity of E- and Z-p-coumaryl fatty acid esters from cv. Annurca apple fruits. J Agric Food Chem 53(9):3525-3529

14. Song JL, Zhu K, Feng X, Zhao X (2015) Protective effect Malus pumila Mill leaf polyphenols in reserpine-induced gastric ulcer in mice. J Korean Soc Appl Biol Chem 58(2):249-256

15. Roemmelt S, Zimmermann N, Rademacher W, Treutter D (2003) Formation of novel flavonoids in apple (Malus $x$ domestica) treated with the 2-oxoglutarate-dependent dioxygenase inhibitor prohexadione-Ca. Phytochemistry 64(3):709-716

16. Wang JM, Lian PL, Yu Q, Wei WJ, Kang WY (2017) Puriication, characterization and procoagulant activity of polysaccharides from Angelica dahurice roots. Chem Cent J 11:17

17. Chen $H$, Jin $M$, Wang $Y F$, Wang YQ, Meng L, Li R, Wang JO, Gao L, Kong Y, Wei JF (2014) Effect of Toona microcarpa harms leaf extract on the coagulation system. Biomed Res Int 2014:22-32

18. Kong Y, Shao Y, Chen H, Ming X, Wang JB, Li ZY, Wei JF (2013) A Novel factor Xa-inhibiting peptide from centipedes venom. Int J Pept Res Ther 19(4):303-311

19. Kim SY, Gao LL, Lee WC, Ryu KS, Lee KR, Kim YC (1999) Antioxidative Fiavonoids from the leaves of Morus alba. Arch Pharmacal Res 22(1):81-85

20. Lim SS, Yu Jung Jung YJ, Hyun SK, Lee YS, Jae Sue Choi JS (2006) Rat lens aldose reductase inhibitory constituents of Nelumbo nucifera stamens. Phytother Res 20(10):825-830
21. Nguelefack TB, Mbakam FHK, Tapondjou LA, Watcho P, Nguelefackmbuyo EP, Ponou BK, Albert Kamanyi A, Park H (2011) A dimeric triterpenoid glycoside and flavonoid glycosides with free radical-scavenging activity isolated from Rubus rigidus var. camerunensis. Arch Pharm Res 34(4):543-550

22. Zhang LQ, Yang XW, Zhang YB, Zhai YY, Xu W, Zhao B, Liu DL, Yu HJ (2012) Biotransformation of phlorizin by human intestinal flora and inhibition of biotransformation products on tyrosinase activity. Food Chem 132:936-942

23. Manguro LOA, Ugi I, Hermann R, Lemmen P (2003) Flavonol and drimane-type sesquiterpene glycosides of Warburgia stuh/mannii leaves. Phytochem 63(4):497-502

24. Yin W, Liu JQ (2016) The flavonoids of chemical constituents of Osmanthus fragrans roots. J Chin Med Mater 39(7):1550-1553

25. Xie PY, Zhang Y, Wang XB, Wei JF, Kang WY (2017) Antithrombotic effect and mechanism of Rubus spp. Blackberry. Food Funct 8(5):2000-2012

26. Moradkhania S, Kobarfard F, Ayatollahi SAM (2014) Phytochemical investigations on chemical constituents of Achillea tenuifolia Lam. Iran J Pharm Res 13(3):1049-1054

27. Bilia AR, Catalano S, Simone FD, DEsimone F, Morelli I, Pizza C (1991) An acetylated flavanone glucoside from leaves of Pyracantha coccinea. Phytochem 30(11):3830-3831

28. Félix-silva J, Souza T, Camara RBJ, Cabral B, Silvajunior AAD, Rebecchi IMM, Zucolotto SM, Rocha HAO, Fernandespedrosa MF (2014) In vitro anticoagulant and antioxidant activities of Jatropha gossypiifolia L. (Euphorbiaceae) leaves. BMC Complement Altern Med 14(1):405

29. Cao PR, Xie PY, Wang XB, Wang JM, Wei JF, Kang WY (2017) Chemical constituents and coagulation activity of Agastache rugosa. BMC Complement Altern Med 17(1):93

30. Mao WJ, Li HY, Li Y, Zhang HJ, Qi XH, Sun HH, Chen Y, Guo SD (2009) Chemical characteristic and anticoagulant activity of the sulfated polysaccharide isolated from Monostroma latissimum (Chlorophyta). Int J Biol Macromol 44(1):70-74

31. Xie PY, Cui LL, Shan Y, Kang WY (2017) Antithrombotic effect and mechanism of radix paeoniae rubra. Biomed Res Int 2017:1-9

32. Yin ZH, Zhang W, Zhang JJ, Kang WY (2017) Isolation, purifcation, structural analysis and coagulatory activity of water-soluble polysaccharides from Ligustrum lucidum Ait fowers. Chem Cent J 11:98

33. Reddy CR, Reddy MD, Dilipkumar U (2014) Total synthesis of a pyrrole lactone alkaloid, longanlactone. Eur J Org Chem 28:6310-6313

34. Reddy CR, Uredi Dilipkumar U, Reddy MD, Rao NN (2013) Total synthesis and revision of the absolute configuration of seimatopolide B. Org Biomol Chem 11(20):3355-3364

35. Reddy MD, Blanton AN, Watkins EB (2017) Palladium-catalyzed, N-(2-Aminophenyl) acetamide-assisted ortho-arylation of substituted benzamides: application to the synthesis of urolithins B, M6, and M7. J Org Chem 82(10):5080-5095

\footnotetext{
Ready to submit your research? Choose BMC and benefit from:

- fast, convenient online submission

- thorough peer review by experienced researchers in your field

- rapid publication on acceptance

- support for research data, including large and complex data types

- gold Open Access which fosters wider collaboration and increased citations

- maximum visibility for your research: over 100M website views per year
}

At BMC, research is always in progress.

Learn more biomedcentral.com/submissions 